\title{
Abnormal Findings in Haemograms of Dachshund Puppies: Presumptive (Immunodeficiency) Familial Disease
}

\author{
${ }^{1}$ Vanessa Turinelli, ${ }^{2}$ Alessandra Gavazza, ${ }^{3}$ Susanne Rushig and ${ }^{2}$ Mario Giorgi \\ ${ }^{1}$ IDEXX Laboratories, Milano, Italy \\ ${ }^{2}$ Department of Veterinary Clinic, Faculty of Veterinary Medicine, University of Pisa, Italy \\ ${ }^{3}$ IDEXX Laboratories, Ludwigsburg, Germany
}

Received 2012-06-01, Revised 2012-06-29; Accepted 2012-08-09

\begin{abstract}
In a litter of seven Dachshund puppies, one subject was stillborn and six presented with diffuse skin infections characterized by dermatitis, abscessation, pustules, crusts and ulcers. Two of the puppies were referred for further evaluation. One male puppy was referred at four months of age and a sister litter mate was referred two months later. A complete blood count, biochemical and histological examination were performed on these puppies to identify the pathologic process. Clinical, biochemical, haematological and histological evaluation of subjects. This report characterizes quantitative and qualitative haematological abnormalities in two puppies that resulted in a diagnosis of dysmyelopoiesis. The existence of a familial immunodeficiency syndrome was speculated. This is the first report of such a syndrome in Dachshund puppies.
\end{abstract}

Keywords: Dysmyelopoiesis, Blood Smear, Neutrophil Function Disorder, Dachshund

\section{INTRODUCTION}

\subsection{Case Presentation}

In a litter of seven Dachshund puppies, one was stillborn and six presented with diffuse skin infection characterized by dermatitis, abscessation, pustules, crusts and ulcers. Two of the puppies were referred for further evaluation.

\subsection{Case 1}

A four-month old male presented with fever, lethargy, anorexia, vomiting, diarrhea, dermatitis and conjunctivitis. A Complete Blood Count (CBC) showed: mild normocytic hypochromic non-regenerative anemia (RBC $4.11 \times 10^{12} / \mathrm{L}, \quad$ Reference Interval (RI) $6-9 \times 10^{12} / \mathrm{L}$; hemoglobin $72 \mathrm{~g} \mathrm{~L}^{-1}$, RI 150-190 $\mathrm{g} \mathrm{L}^{-1}$; hematocrit $26 \%$, RI 38-55\%; MCV $63 \mathrm{fL}$, RI 60-77 fL; MCHC $28 \mathrm{~g} \mathrm{dL}^{-1}$, RI 31-34 $\mathrm{g} \mathrm{dL}^{-1}$; reticulocytes $41400 \mu \mathrm{L}^{-1} \mathrm{RI}>60.000$ ), mild leukocytosis $\left(15.6 \times 10^{9} \mathrm{~L}^{-1}\right.$, RI $\left.6-12 \times 10^{9} \mathrm{~L}^{-1}\right)$ and mild thrombocytopenia $\left(113 \times 10^{9} \mathrm{~L}^{-1}\right.$, RI $150-500 \times 10^{9}$ $\left.\mathrm{L}^{-1}\right)$. The differential leukocyte count was: segmented neutrophils $2652 \mu \mathrm{L}^{-1}$ RI $3000-10000 \mu \mathrm{L}^{-1}$, eosinophils
$624 \mu \mathrm{L}^{-1}$ RI 0-600 $\mu \mathrm{L}^{-1}$, lymphocytes $3900 \mu \mathrm{L}^{-1} \mathrm{RI}$ $1000-4000 \mu \mathrm{L}^{-1}$ and atypical cells $8586 \mu \mathrm{L}^{-1} \mathrm{RI} 0 \mu \mathrm{L}^{-1}$. Microscopic evaluation of the blood smear revealed the presence of keratocytes, schistocytes, anulocytes and severe signs of dysplasia in both neutrophils and monocytes. The dysp lasia was characterized by abnormal nuclear shape, hypersegmented and/or hyposegmented nuclei, irregular chromatin pattern and atypical cytoplasmic granules. The platelets were dysplastic to an extent that they were initially mistaken for blood parasites, because most of them had a tail-like structure (Fig. 1 and 2). Clinical signs of altered primary hemostasis were not evident. For this reason, PCR screening for Trypanosoma spp. was performed, but it was negative. Cytological diagnosis of dysmyelopoiesis was made.

The biochemistry panel was characterized by mild hypoalbuminemia (25 $\mathrm{g} \mathrm{L}^{-1}$ RI 32-46 $\mathrm{g} \mathrm{L}^{-1}$ ) and mild hyperglobulinemia (38 $\mathrm{g} \mathrm{L}^{-1}$ RI $15-35 \mathrm{~g} \mathrm{~L}^{-1}$ ) with increase in acute phase protein fractions and a mild increase in alkaline phosphatase $\left(364 \mathrm{U} \mathrm{L}^{-1} \mathrm{RI}<81 \mathrm{~g}\right.$ $\left.\mathrm{L}^{-1}\right)$ and creatine phosphokinase $\left(526 \mathrm{U} \mathrm{L}^{-1} \mathrm{RI}<180 \mathrm{U}\right.$ $\mathrm{L}^{-1}$ ). The mild hypoalbuminemia and hyperglobulinemia Corresponding Author: Mario Giorgi, Department of Veterinary Clinic, Faculty of Veterinary Medicine, University of Pisa, Italy 
with increase in acute phase protein fractions was attributed to the persistent infection.

The dog died three days after referral and before bone marrow sampling could be attempted. Necropsy and histopathological examinations were carried out and the results showed: multifocal, necrotizing to suppurative hepatitis, neutrophilic lymphadenitis of the mesenteric lymph nodes, ulcerative enteritis of the small intestine and extramedullary hematopoiesis in the spleen. The histological findings were suggestive of septicemia.

\subsection{Case 2}

A six-month-old female presented with fever, lethargy, anorexia, vomiting, diarrhea and diffuse skin granuloma. The $\mathrm{CBC}$ showed: mild normocytic, hypochromic non regenerative anemia (RBC $4.04 \times 10^{12}$ $\mathrm{L}^{-1}$, RI 6-9×10 ${ }^{12} \mathrm{~L}^{-1}$; hemoglobin $82 \mathrm{~g} \mathrm{~L}^{-1}$, RI 150-190 g $\mathrm{L}^{-1}$; hematocrit 31\%, RI 38-55\%; MCV $76 \mathrm{fL}$, RI 60-77 fL; MCHC $26 \mathrm{~g} \mathrm{dL}^{-1}$, RI 31-34 $\mathrm{g} \mathrm{dL}^{-1}$; reticulocytes $38000 \mu \mathrm{L}^{-1}$, RI $>60.000$ ) and moderate leukocytosis $\left(35.3 \times 10^{9} \mathrm{~L}^{-1}\right.$, RI $\left.6-12 \times 10^{9} \mathrm{~L}^{-1}\right)$. The platelet count was normal $\left(210 \times 10^{9} \mathrm{~L}^{-1}, \quad\right.$ RI $\left.150-500 \times 10^{9} \mathrm{~L}^{-1}\right)$. The differential leukocyte count was: eosinophils $706 \mu \mathrm{L}^{-1}$ RI 0-600 $\mu \mathrm{L}^{-1}$, lymphocytes $10590 \mu \mathrm{L}^{-1}$ RI 1000-4000 $\mu^{-1} \mathrm{~L}$, atypical myelo-monocyte cells $22592 \mu \mathrm{L}^{-1}$ RI 0 $\mu \mathrm{L}^{-1}$ and blasts $1412 \mu \mathrm{L}^{-1}$ RI $0 \mu \mathrm{L}^{-1}$. The atypical myelo-monocytic cells were characterized by a large size, abnormal nuclear shape, irregular clumped chromatin, rarely visible nucleoli and a large amount of cytoplasm, often containing atypical granules (Fig. 3). Blasts were characterized by medium or large size, nucleus round and convoluted, irregular granular chromatin, one or more nucleoli and large amount of basophilic cytoplasm. The platelets were often very dysplastic (Fig. 4). It is important to emphasize that even though a severe platelets dysplasia was noted, no specific clinical signs such as petechiae were observed. This suggests that the morphological alterations did not have a significant impact on function. The cytologic interpretation suggested a dysmyelopoiesis syndrome.

The biochemistry panel was characterized by mild hypoalbuminemia ( $\left.28 \mathrm{~g} \mathrm{~L}^{-1} \mathrm{RI} 32-46 \mathrm{~g} \mathrm{~L}^{-1}\right)$ and mild hyperglobulinemia (45 $\mathrm{g} \mathrm{L}^{-1}$ RI 15-35 $\mathrm{g} \mathrm{L}^{-1}$ ) with increase in acute phase protein fractions.

As the dog's clinical condition continued to worsen, she was euthanized at 8 month of age.

Unfortunately, permission for cytological examination of the bone marrow could not be obtained. This prevented the definitive diagnosis of a dysmyelopoiesis.

Further information on the other litter mates was supplied by the referring practitioner.

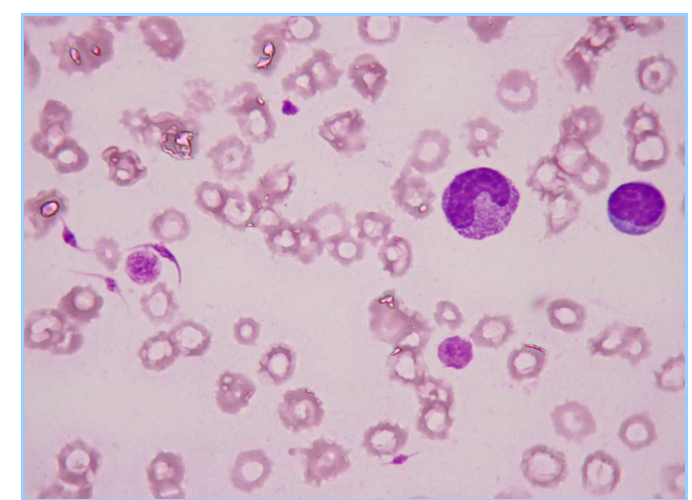

Fig. 1. Case 1. Dysplastic granulocytes with hyposegmented nucleus and abnormal granules in the cytoplasm and atypical "tailed" platelets. May Grunwald Giemsa stain (100x)

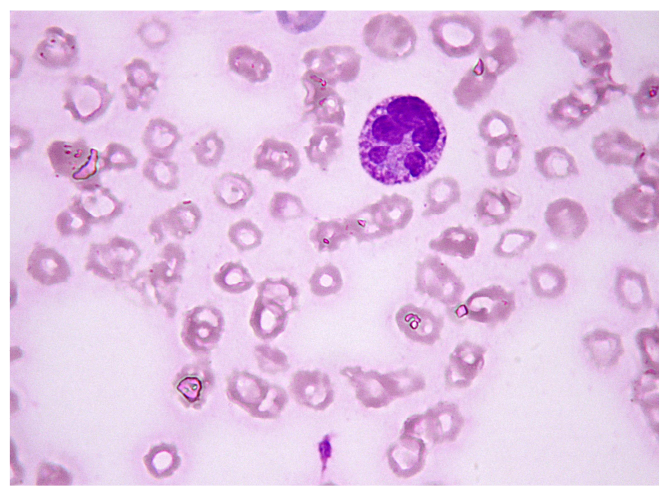

Fig. 2. Case 1. Hypersegmented granulocytes neuthrophils with numerous abnormal granules in the cytoplasm. May Grunwald Giemsa stain (100x)

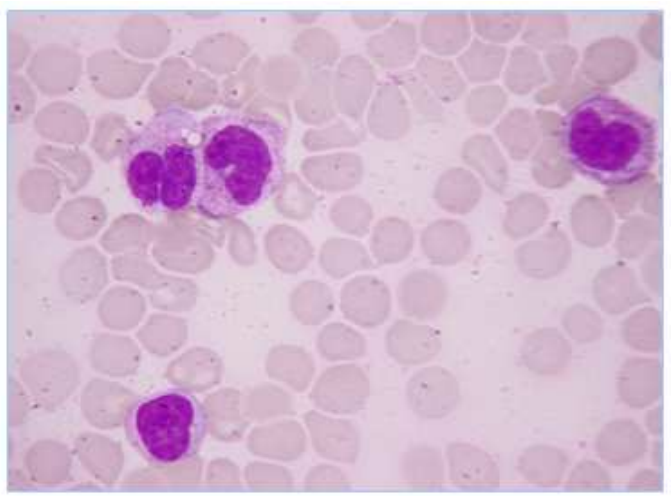

Fig. 3. Case 2. Large abnormal myelo-monocytic cells with abnormal nuclear shape, irregular clumped chromatin, rarely visible nucleoli and large amount of cytoplasm that contains atypical granules. May Grunwald Giemsa stain (100x) 


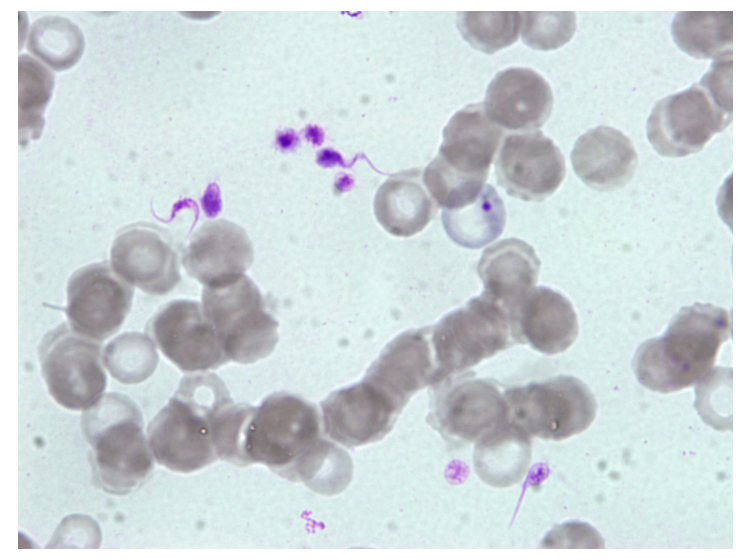

Fig. 4. Case 2. Atypical platelet characterized by the presence of "tail" and resembling blood parasites. May Grunwald Giemsa stain (100x)

\subsection{Case 3-4}

Another puppy from the same litter presented with similar skin lesions and died four months after birth. A diagnosis of peritonitis and suppurative mesenteric lymphadenitis was made following necropsy. Another puppy also presented with similar skin lesions in addition to a severe diarrhea. She was euthanized at five-months of age and numerous abscesses were found in the liver and mesenteric lymph nodes during necropsy.

\subsection{Case 5-6}

These subjects presented with similar clinical symptoms but died before they could be referred to the hospital.

\section{DISCUSSION}

Dysmyelopoiesis is defined as a hematologic disorder characterized by the presence of morphologic abnormalities (i.e., dysplasia) in one or more hematologic cell lines in the blood or bone marrow (Weiss, 2006; Weiss and Wardrop, 2011). Dysmyelopoieses are divided into myelodysplastic syndromes, secondary dysmyelopoiesis and congenital dysmyelopoiesis. Causes of dysmyelopoiesis include acquired mutations in hematopoietic stem cells (i.e., myelodysplastic syndromes), congenital defects in hematopoiesis and dysmyelopoietic conditions associated with various disease processes, drug treatments, or toxin exposure (Weiss, 2005). The congenital forms, causing functional defects, have been well described in some canine breeds: e.g., Canine
Leucocytes Adhesion Deficiency (CLAD) in Irish Setter and Irish setter-cross-bred, chronic granulomatous disease in Dobermann Pinschers, myeloperoxidase deficiency in Gray Collies, altered chemiluminescence in Weimaraners and Pelger-Huet anomaly (Weiss and Wardrop, 2011).

CLAD was initially described in a male Irish setter by Renshaw et al. (1975) and collegues, they called the condition "Canine granulocitopathy syndrome". The dogs had a clinical history of recurrent infections associated with pyrexia and neutrophilia and circulating leukocytes isolated from the $\operatorname{dog}$ at 5 months of age showed a bactericidal defect due to abnormal expression of the CD11b/CD18 complex (Bauer et al., 2004; Gu et al., 2004). The puppies described in this report showed severe skin lesions and septicemia, both phenomenons can be attributed to a neutrophil hypofunction. Neutrophils are essential to preventing bacterial infection and dogs with naturally occurring septic and non-septic inflammatory diseases had an increased cell-expression of CD11b (Weiss and Wardrop, 2011). Hence, we might speculate that they were affected by a Canine granulocitopathy syndrome.

A single case of cabot ring cells has been reported in the Dachshund. This was characterized as a severe dyserythropoiesis in an 11-year-old female that presented with depression, diarrhea, weight loss and radiographic evidence of abdominal masses (Lukaszewska and Lewandowski, 2008). Examination of the peripheral blood smear revealed erythroid dysplasia, anisocytosis with macrocytosis, Howell Jolly bodies, atypical nucleated cells, neutrophil hypersegmentation and the presence of giant forms. The final diagnosis was myelodysplasia secondary to metastatic adenocarcinoma (Lukaszewska and Lewandowski, 2008).

A review on Pneumocystis carinii in the miniature Dachshund reported four cases with hyperpnoea, tachypnoea, exercise intolerance and signs suggestive of immune incompetence. It is probable that Pneumocystis carinii infection in these cases was the result of an immunodeficiency (Lobetti et al., 1996).

The hematological findings of both leukocytosis and severe leukocytic dysplasia is suggestive of an abnormal function of the bactericidal cells. Tests on neutrophil function were not performed in these cases because it was not possible to access the appropriate diagnostic tests in Italy. However, both the morphological cellular abnormalities and the clinical symptoms are suggestive of reduced cell function and consequently, a functional defect of the immune system. The death of other four puppies supports the hypothesis of a familial immunodeficiency. 


\section{CONCLUSION}

This is the first report describing a familial imunodeficiency syndrome in Dacschund dogs characterized by significant hematological changes, particularly of the leukocyte cells

\section{ACKNOWLEDGEMENT}

Researchers acknowledged to Dr E. Owen (University of Queensland, Australia) the both the scientific and English editing of the manuscript.

\section{REFERENCES}

Bauer, T.R. Jr., Y.C. Gu, K.E. Creevy, L.M. Tuschong and L. Embree et al., 2004. Leukocyte adhesion deficiency in children and Irish setter dogs. Pediatr. Res., 55: 363-367. PMID: 14711903

Gu, Y.C., T.R. Bauer Jr., M.R. Ackermann, C.W. Smith Jr. and M.E. Kehrli Jr. et al., 2004. The genetic immunodeficiency disease, leukocyte adhesion deficiency, in humans, dogs, cattle and mice. Comput. Med., 54: 363-372. PMID: 15357315
Lobetti, R.G., A.L. Leisewitz and J.A. Spencer, 1996. Pneumocystis carinii in the miniature dachshund: case report and literature review. J. Small Anim. Pract., 37: 280-285. PMID: 8965482

Lukaszewska, J. and K. Lewandowski, 2008. Cabot rings as a result of severe dyserythropoiesis in a dog. Vet. Clin. Pathol., 37: 180-183. PMID: 18533917

Renshaw, H.W., C. Chatburn, G.M. Bryan, R.C. Bartsch and W.C. Davis, 1975. Canine granulocytopathy syndrome: Neutrophil dysfunction in a dog with recurrent infections. J. Am. Vet. Med. Assoc., 166: 443-447. PMID: 1089620

Weiss, D.J. and K.J. Wardrop, 2011. Schalm's Veterinary Hematology. 6th Edn., John Wiley and Sons, USA., ISBN-10: 047096183X, pp: 1232.

Weiss, D.J., 2005. Recognition and classification of dysmyelopoiesis in the dog: A review. J. Vet. Intern. Med., 19: 147-154. PMID: 15822557

Weiss, D.J., 2006. A retrospective study of the incidence and the classification of bone marrow disorders in the dog at a veterinary teaching hospital (19962004). J. Vet. Intern. Med., 20: 955-961. PMID: 16955822 